\title{
THE SIZE DISTRIBUTIONS OF FRAGMENTS EJECTED AT A GIVEN VELOCITY FROM IMPACT CRATERS
}

\author{
John D. O'Keefe and Thomas J. Ahrens \\ Seismological Laboratory 252-21 \\ California Institute of Technology \\ Pasadena, CA 91125 \\ April 2, 1986
}

The mass distribution of fragments that are ejected at a given velocity for impact craters is modeled to allow extrapolation of laboratory, field, and numerical results to large-scale planetary events. The model is semi-empirical in nature and is derived from (1) numerical calculations of cratering and the resultant mass versus ejection velocity, (2) observed ejecta blanket particle size distributions, (3) an empirical relationship between maximum ejecta fragment size and crater diameter, (4) measurements and theory of maximum ejecta size versus ejecta velocity, and (5) an assumption on the functional form for the distribution of fragments ejected at a given velocity. This model implies that for planetary impacts in to competent rock, the distribution of fragments ejected at a given velocity is broad, e.g. $68 \%$ of the mass of the ejecta at a given velocity contains fragments having a mass less than 0.1 times a mass of the largest fragment moving at that velocity. The broad distribution suggests that in impact processes, additional comminution of ejecta occurs after the upward initial shock has passed in the process of the ejecta velocity vector rotating from an initially downward orientation. This additional comminution produces the broader size distribution in impact ejecta as compared to that obtained in simple brittle failure experiments.

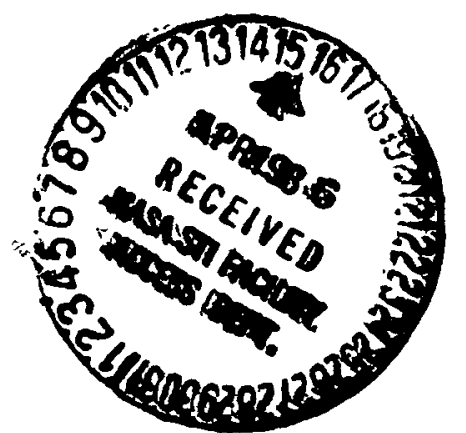

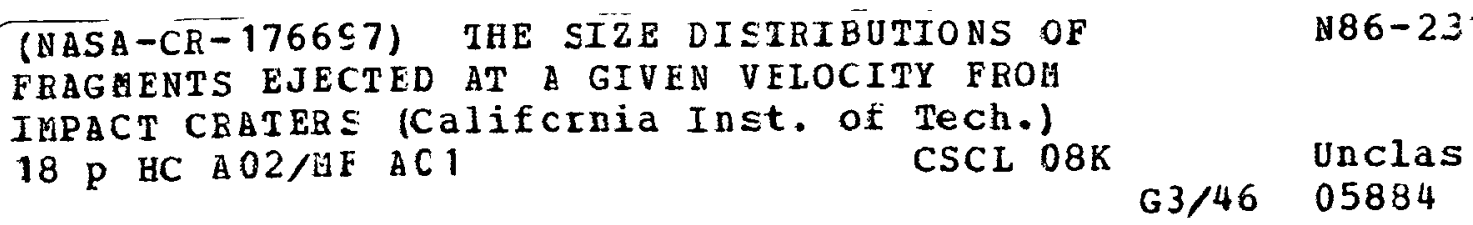




\subsection{INTRODUCTION}

Meteorites come from collisions on objects varying in size from asteroidal to terrestrial planets ${ }^{1-8}$. Massive extinctions on the earth may have resulted from large impacts ${ }^{9}$. The origin of meteorites is dependent on the maximum-size fragment that can be ejected at velocities exceeding the planetary escape velocity, whereas the biological consequence of large impacts is dependent on the number of mass of fine particles (diameters $\lesssim 10^{-6}$ m) that are ejected high in to the atmosphere. ${ }^{10}$

A model was developed for the mass distributions of fragments that are ejected at a given velocity for impact events ${ }^{10}$. The model is semi-empirical in approach and is consistent with empirical data from small scale impact experiments, nuclear, and chemical explosion data, ejecta fragment size vs. velocity data, largest ejecta fragment size vs. crater size data and total mass ejected vs. impact energy. In addition, the model is based upon calculations of mass ejected as a function of ejection velocity and maximum fragment size as a function of strain rate models. Using the above data base, the distribution function for the fragments was derived. In this paper we have extended our previous approach and have found additional data that strengthen those conclusions.

\subsection{CUMULATIVE MASS DISTRIBUTION OF FRAGMENTS}

The objective of this paper is to determine the distribution of fragments that are ejected at a given velocity during an impact event. Shown in figure 1 is a typical impact-induced flow field at a given time. The figure demonstrates the decrease in ejecta velocity, $V$, with decreasing radius, $r$, given approximately by $\mathrm{V} \propto \mathrm{r}^{-3}$. The crater plume consists of particles ejected at positions $r$ at times $t$ and the velocity of ejection varies as a function of position along the surface. Thus the plume necessarily changes shape during its evolution.

By definition, the cumulative amount of mass $M_{c}$ of fragments ejected at all velocities of mass greater than $m$ is given by

$$
M_{c} \equiv \int_{v_{m i n}}^{v} \frac{\partial M_{c v}}{\partial V} f\left(m, m_{b v}(V)\right) d V
$$

where $\frac{\partial M_{c v}}{\partial V}$ is the amount of mass ejected at velocities between $V$ and $V+d V$, and $f\left(m, m_{b v}(V)\right)$ is the unknown distribution of fragments ejected at $V$. The expression for 
the amount of mass ejected at a given velocity, $M_{c v}$, can be determined from the fits to the cumulative amount of mass ejected at velocities greater than $\mathrm{V}$ obtained either from theoretical calculations ${ }^{10-13}$ (see figure 2) or experimental data ${ }^{14-15}$.

$$
\frac{\partial \mathrm{M}_{\mathrm{cv}}}{\partial \mathrm{V}}=\frac{\xi \mathrm{M}_{\mathrm{T}}}{\mathrm{V}_{\min }}\left[\frac{\mathrm{V}}{\mathrm{V}_{\min }}\right]^{-(\xi+1)} \quad \mathrm{V}_{\min } \leq \mathrm{V} \leq \mathrm{V}_{\mathrm{MAX}}
$$

where $M_{T}$ is the total amount of mass ejected, and $V_{\min }$ and $V_{\max }$ are the minimum and maximum velocities of ejection of solid particles. A discussion of the thermodynamic constraints on the maximum velocity of ejection is given by O'Keefe and Ahrens ${ }^{10}$. The exponent $\xi$ is related to the mass scaling parameter ${ }^{16}, \alpha$,

$$
\text { by } \xi=6 \alpha /(\alpha-3)
$$

The mass scaling parameter specifies the relation between $M_{c}$ and $m_{b}$, the maximum fragment mass.

$$
\frac{\mathrm{M}_{\mathrm{c}}}{\mathrm{M}_{\mathrm{T}}}=\left[1-\left(\mathrm{m} / \mathrm{m}_{\mathrm{b}}\right)^{\alpha / 3}\right]
$$

The minimum velocity of ejection varies with the planetary strength at small scales and $\mathrm{Rg}$ at large scales, where $\mathrm{R}$ is the size of the impactor and $\mathrm{g}$ is the planetary gravity.

The key assumption in the theory is that the functional form of the distribution of fragments ejected at a given velocity is similar to the distribution function of the fragments in the ejecta blanket. For explosive events Jaeger et al. ${ }^{17}$ have found that this is the case. With this assumption the cumulative fraction of mass fragments of mass greater than $\mathrm{m}$ is given by

$$
\mathrm{f}\left(\mathrm{m}, \mathrm{m}_{\mathrm{bv}}(\mathrm{V})\right)=\left[1-\left(\mathrm{m} / \mathrm{m}_{\mathrm{bv}}\right)^{\frac{\beta}{3}}\right]
$$

where $m_{b v}$ is the mass of the largest fragment ejected at a given velocity.

In addition, we assume that the mass of the largest fragment ejected at a given velocity is a function of the velocity of ejection and is given by

$$
\frac{\mathrm{m}_{\mathrm{bv}}(\mathrm{V})}{\mathrm{m}_{\mathrm{b}}}=\left(\mathrm{V} / \mathrm{V}_{\min }\right)^{-\delta}
$$

where $\delta$ is an unknown parameter to be determined 
The above functional form can be justified by comparison to explosion cratering data $^{18}$, planetary cratering observational analysis ${ }^{19}$, and theoretical considerations. Ejecta velocities were measured on a 100 ton TNT above ground explosion called Middle Gust III, and are summarized by Schoutens ${ }^{18}$. The size-dependent ejection velocity limit inferred from film recording and analysis by Wisotski ${ }^{20}$ and corrections for deceleration in air $^{21}$ is shown in figure 3 . The range of launch velocities correlates with $\delta \cong 3$.

The theory developed by Grady and Kipp ${ }^{22}$ can be used to determine an expression for $\delta$. They found that the mass of the largest fragment produced is a function of the strain-rate

$$
\mathrm{m}_{\mathrm{b}} \propto \dot{\epsilon}^{3 l /(l+3)}
$$

where $l \cong 8$ for rocks.

The strain-rate is related to the velocity field by

$$
\dot{\epsilon} \propto \frac{\partial \mathrm{V}}{\partial \mathrm{r}}
$$

where $r$ is, again, the surface radial coordinate. The surface velocity field has the following form for impacts ${ }^{16}$.

$$
V \propto\left(\frac{r}{R}\right)^{-3 / \xi}
$$

where $R$ is the radius of the impactor. Evaluating the surface strain-rate, the mass of the largest fragment created at a given velocity is

$$
\mathrm{m}_{\mathrm{b}} \propto\left(\frac{\mathrm{V}}{\mathrm{V}_{\min }}\right)^{\frac{(\xi+3) l}{l+3}}
$$

and thus

$$
\delta=(\xi+3) l /(l+3)
$$

For a variety of materials, $\delta$ ranges from 3 to 3.6. The expression given by equation 12 for cumulative mass distribution of fragments in the ejecta blankets is compared to experimental data in figure 6. In the case shown, $\delta=3$ and $\xi=1.4$. The only unknown parameter in equation 12 is $\beta$, which is in the exponent in mass distribution of fragments ejected at a given velocity. Referring to figure 6, we get good agreement with the fragment size data when $\beta \cong 0.5$. Note that this has the same value as the 
cumulative mass distribution exponent $\alpha$ (Eq. 6). This means that the distribution of fragments ejected at a given velocity is polydispersed and that in the flow and ejection process, grinding and crushing has occurred since fracture processes are more monodispersed $^{22,26}$.

The integral for the cumulative mass distribution in the ejecta blanket can be evaluated by substituting equations 2,5 , and 6 in equation 1 , which yields the following

$$
\begin{array}{rlrl}
\frac{M_{c}}{M_{T}}=\left[1-\left(\frac{m}{m_{b}}\right)^{+\frac{\xi}{\delta}}\right]+\left(1-\frac{\delta \beta}{3 \xi}\right)^{-1}\left[\left(\frac{m}{m_{b}}\right)^{+\frac{\xi}{\delta}}-\left(\frac{m}{m_{b}}\right)^{\frac{\beta}{3}}\right] & \text { for } m_{2} m_{s} \\
& =0 & \text { for } m<m_{s}
\end{array}
$$

where $\mathrm{m}_{\mathrm{s}}$ is the mass of the smallest fragments. Note that in the case of both impact craters $^{23}$ and explosion events ${ }^{17}$ that there is a characteristic scale size in which the number of smaller fragments decreases.

The above expression (equation 12) can be compared to experimental data on the cumulative mass distributions fragments ejected from impact craters ${ }^{23-25}$. Both impact and explosion fragments distributions can be described by expressions of the following for $\mathrm{m}^{11,26,27}$.

$$
\mathrm{M}_{\mathrm{c}} / \mathrm{M}_{\mathrm{T}}=\left[1-\left(\mathrm{m} / \mathrm{m}_{\mathrm{b}}\right)^{\alpha / 3}\right]
$$

where $m_{b}$ was found by Gault et al. ${ }^{23}$ to correlate with the total mass ejected from the crater for large range of crater sizes. Shown in figure 5 is that data which is described by

$$
\mathrm{m}_{\mathrm{b}}=0.2 \mathrm{M}_{\mathrm{T}}^{0.8}
$$

The following is an expression for the mass fraction of fragments that are ejected with velocities in fraction range $\Delta \mathrm{V}$ at $\mathrm{V}$.

$$
\frac{\Delta \mathrm{M}_{\mathrm{cv}}}{\mathrm{M}_{\mathrm{T}}}=\xi\left(\frac{\Delta \mathrm{V}}{\mathrm{V}_{\min }}\right)\left(\frac{\mathrm{V}}{\mathrm{V}_{\min }}\right)^{-(\xi+1)}\left[1-\left(\frac{\mathrm{m}}{\mathrm{m}_{\mathrm{b}}}\right)^{\frac{\alpha}{3}}\left(\frac{\mathrm{V}}{\mathrm{V}_{\min }}\right)^{\frac{\delta \alpha}{3}}\right]
$$




\subsection{CONCLUSIONS}

1. The ejecta velocity, $V$, of surface material achieved upon impact of a projectile of radius, $R$, at a radius, $r$, is approximately given by $V \propto(R / r)^{3}$.

2. The cumulative mass of impact ejecta launched at a given velocity is described by a power law function, in which the exponent $\xi$ is related to the exponent, $\alpha$, of the power law function which describes the cumulate mass, versus, mass greater or equal to a given value, of the total ejecta distribution by $\xi=6 \alpha /(\alpha-3)$.

3. The velocity of the largest fragments in a given cratering ejecta distribution is described by a power law function of ejecta velocity where the scaling exponent, $\delta$, is given by $\delta=(\xi+3) l /(l+3)$ where $l$ is the parameter in the expression for the largest fragment $m_{b}$ produced upon brittle failure at varying strain rates as described by Grady and Kipp ${ }^{22}$. The most massive fragment launched at a given velocity, $m_{b}$, is found to depend on velocity as $V, m_{b} \propto V^{-3}$, in agreement with results of Vickery ${ }^{19}$.

4. Both the cumulative mass of ejecta and the mass of ejecta launched to a velocity, $V$, and the total mass greater than $m$ in the ejecta have similar power law distribution.

5. Brittle fragments distributions obtained in simple failure experiments are very monodispersed, as to their size, in comparison to impact ejecta distributions which are very broadly dispersed with mass to sizes traveling at a given velocity. We infer that additional comminution occurs during impact cratering, presumably during the processes of ejecta rotation which take place on the particle velocity impacted by the shock and changes its direction from being primarily downward directed to achieving a net outward and upward motion.

\section{Acknowledgments:}

Research supported under NASA Grant NSG7129. We appreciate the computational assistance of Michael Lainhart and receiving preprints from Ann Vickery. Contribution number 4325, Division of Geological and Planetary Sciences, California Institute of Technology, Pasadena, California 91125. 


\section{References}

1 Ostertag, R. and Ryder, C. (1983). ALHA 81005: Petrography, shock, Moon, Mars, Giordano Bruno, and composition (abstr.) in Lunar and Planetary Science $X I V$, Lunar and Planetary Institute, 23-24.

2 Bischoff, A. and Stöfler, D. (1985). Clast population statistics of the lunar meteorite Yamato 791197 - Sample from a new source region of the lunar highlands [abs.], Lunar and Planetary Science XVI, 63-64.

3 Bogard, D. D. and Johnson, P. (1983). Martian gases in an Antarctic meteorite. Science, 221, 6.51-653.

4 Wasson, J. T. and Wetherill, G. W. (1979). Dynamical, chemical, and isotopic evidence regarding the formation location of asteroids and meteorites, in Asteroids, $\mathrm{T}$. Gehrels, ed., 926-974, U. Ariz. Press.

5 Melosh, H. J. (1984). Impact ejection, spallation, and the origin of the meteorites, Icarus, 59, 234-260.

6 McSween, H. Y., Jr. (1985). SNC meteorites: Clues to Martian petrologic evolution?, Rev. Geophysics, 23, 391-415.

7 Ott, U. and Begemann, F. (1985). Are all the 'martian' meteorites from Mars? Nature, 317, 509-512.

8 Nyquist, L. E. (1982). Do oblique impacts produce martian meteorites? Proc. Lunar Planet. Sci. Conf. 19th, Suppl. J. Geophys. Res., 88, A779-784.

9 Alvarez, L. E., Alvarez, W., Asaro, F., and Michel, H. V. (1980). Extraterrestrial cause for the Cretaceous-Tertiary extinction. Science, 208, 1095-1108.

10 O'Keefe, J. D. and Ahrens, T. J. (1982). The interaction of the CretaceousTertiary extinction bolide with the atmosphere, ocean, and solid earth. Proc. Conf. on Large Body Impacts and Terrestrial Evolution, Sp. Paper \#190 Geol. Soc. Am., 103-120.

11 O'Keefe, J. D. and Ahrens, T. J. (1977a). Impact induced energy partitioning, melting, and vaporization on terrestrial planets. Proc. Lunar Sci. Conf., 8th, $3357-3374$.

12 O'Keefe, J. D. and Ahrens, T. J. (1977b). Meteorite impact ejecta: Dependence of mass and energy lost on planetary escape velocity. Science, 198, 1249-1251. 
13 O'Keefe, J. D. and Ahrens, T. J. (1986). Oblique impact: A process for providing meteorite samples of other planets, submitted for publication.

14 Stöfller, D., Gault, D. E., Wedekind, J., and Polkowski, G. (1975). Experimental hypervelocity impact in to quartz sand: Distribution and shock metamorphism of ejecta. J. Geophys. Res., 80, 4062-4077.

15 Andrews, R. J. (1975). Origin and distribution of ejecta from near-surface laboratory-scale cratering experiments, Report AFWL-TR-74-314, 207 pp.

16 Housen, K. R., Schmidt, R. M., and Holsapple, K. A. (1983). Crater ejecta scaling laws: Fundamental forms based on dimensional analysis, J. Geophys. Res., 88, 2485-2499.

17 Jaeger, Z, Englman, R., and Slotky, D. (1986). Fractal behaviour and velocitydependence related to spatial variation of fragment sizes, Proc. Int. Symp. on Fracture and Fragmentation, Jerusalem, Jan. 5-8, 1986.

18 Schoutens, J. E. (1979). Nuclear Geophysics Sourcebook, IV-Part II, , Empirical analysis of nuclear and high-explosive cratering and ejecta, Rep. DNA 65 01H-4-2, Defense Nuclear Agency, Washington, D.C.

19 Vickery, A. M. (1986). Size-velocity distribution of large ejecta fragments, ICARUS, submitted.

20 Wisotski, J. (1975). Middle Gust dynamic ejecta measurements, Denver Research Institute, Denver, CO. AFWL-TR-75-104. Air Force Weapons Laboratory, Kirtland Air Force Base, N. M.

21 Seebaugh, W. R. (1975). Science Applications, Inc.: DNA Report 3640 I.

22 Grady, D. E. and Kipp, E. (1980). Continuum modeling of explosive fracture in oil shale. Inst. J. Rock Mech. Min. Sci. \& Geomech. Abstr., 17, 147-157.

23 Gault, D. E., Shoemaker, E. M., and Moore, H. J. (1963). Spray ejected from the lunar surface by meteoroid impact: NASA Publication TND-1767, p. 39.

24 Lange, M. A. and Ahrens, T. J. (1981). Fragmentation of ice by low velocity impacts, in Proc. Lunar \& Planet. Sci. Conf. 12th, Pergamon Press, New York, pp. 1667-1687.

25 Fujiwara, A., Kamimoto, G., and Tsukamoto, A. (1977). Destruction of basaltic bodies by high-velocity impact. ICARUS, 31, 277-288. 
26 Hartmann, W. K. (1969). Terrestrial, lunar, and interplanetary rock fragmentation. ICARUS, 10, 201-203.

27 O'Keefe, J. D. and Ahrens, T. J., 1985. Impact and explosion crater ejecta, fragment size, and velocity, ICARUS, 62, 328-338. 


\section{Figure Captions}

Fig. 1. Cratering flow field at a time, $t$, given by $d / V t=2.0$, where $V$ is impact velocity and $d$ is projectile diameter for water projectile striking silicate half space at $5 \mathrm{~km} / \mathrm{sec}$. The relationship between the ejecta plume and surface velocity field is indicated. The velocity, $\mathrm{V}$, of each fragment of ejecta is approximately related to its take-off radius, $r$, by $V \propto(R / r)^{3}$, where $R$ is the projectile radius.

Fig. 2. Cumulative ejecta mass at velocity less than V versus ejecta velocity. Calculations for impacts of solid silicate and ice at $5 \mathrm{~km} / \mathrm{sec}$ are indicated. Here $\mathrm{M}_{\mathrm{c}} / \mathrm{M}_{\mathrm{T}}=\mathrm{M}_{\mathrm{c}}^{\prime} / \mathrm{M}_{\mathrm{T}^{-1}}$.

Fig. 3. Observed ejecta velocity, versus, ejecta diameter, A, 5 to 10 sec after explosion of MIDDLE GUST III, a 100 ton TNT surface explosion conducted over partially saturated Pierre shale ${ }^{18}$. Size dependent ejecta velocity limit correspond to $\delta=3.0$.

Fig. 4. Ejecta fragment size versus ejection velocity inferred from gravity scaling from Lunar (a) and (b), and Martian (c) craters ${ }^{19}$ mass of fragments correlates with $\delta=3$ for relation $\mathrm{m}_{\mathrm{b}} \propto \mathrm{V}^{-3}$ shown.

Fig. 5. Mass of the largest fragment versus total ejected mass for explosive and impact cratering events ${ }^{23}$.

Fig. 6. Cumulative mass $M_{c}$ (ejected at velocity $V$ ) normalized by the total mass $M_{T}$ (ejected at velocity $\mathrm{V}$ ) versus fragment size normalized to the largest fragment ejected at velocity $V$. Data is for a value of $\xi=1.9$ as indicated and theoretical curves are present for $\beta=0.4,0.5$, and 0.6. Here $M_{c} / M_{T}=M_{c}{ }^{\prime} / M_{T^{-1}}$. 


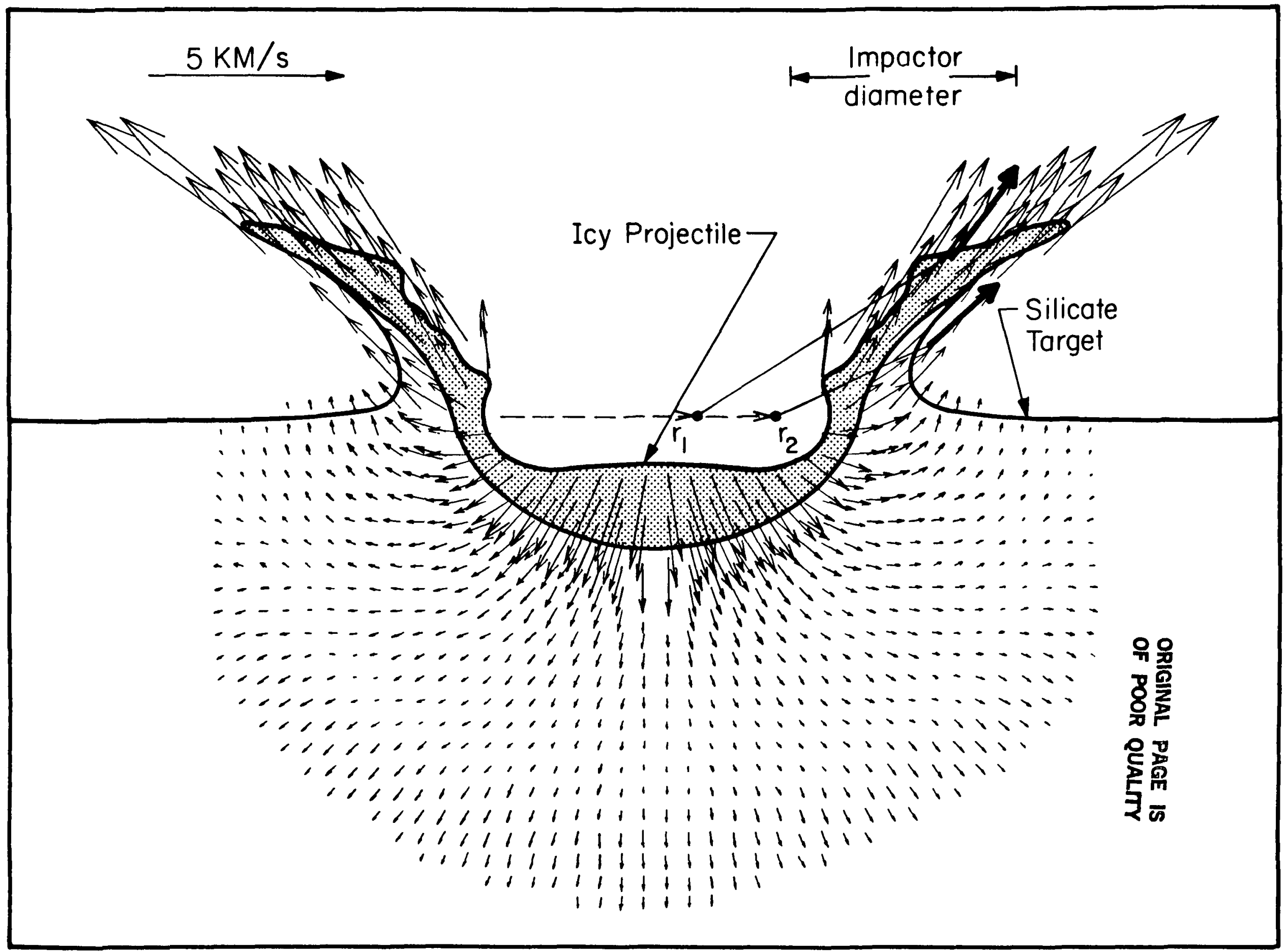




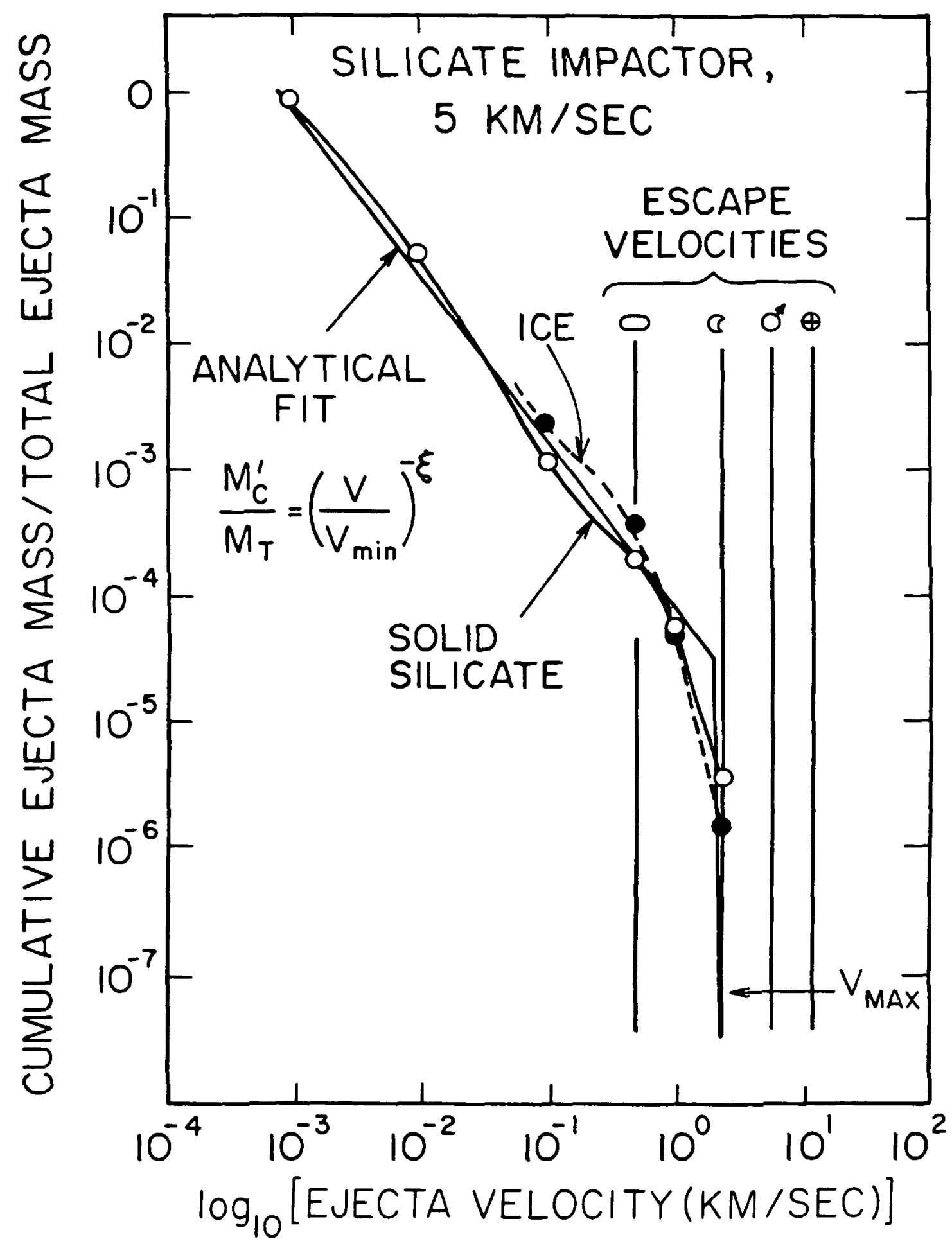




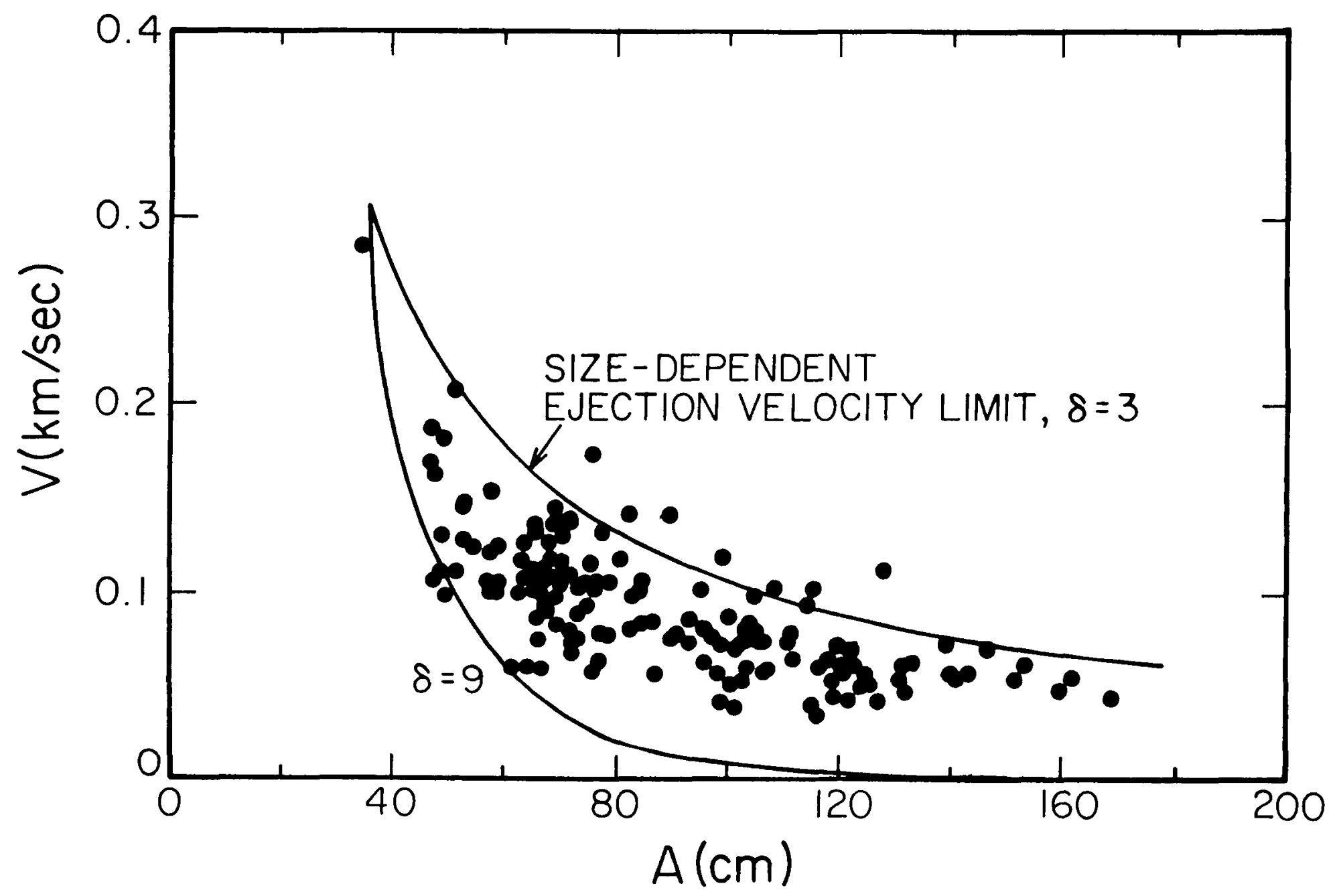




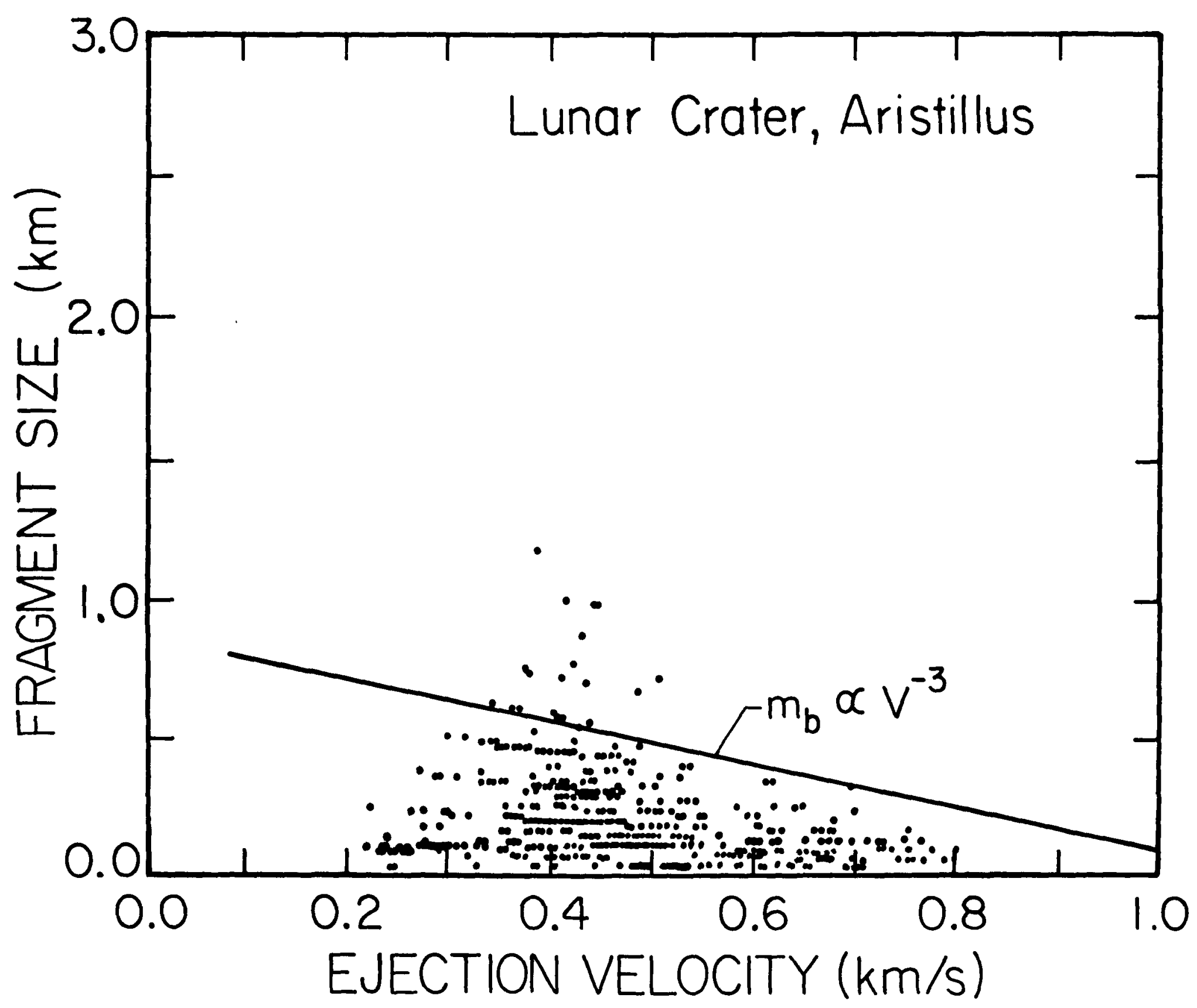




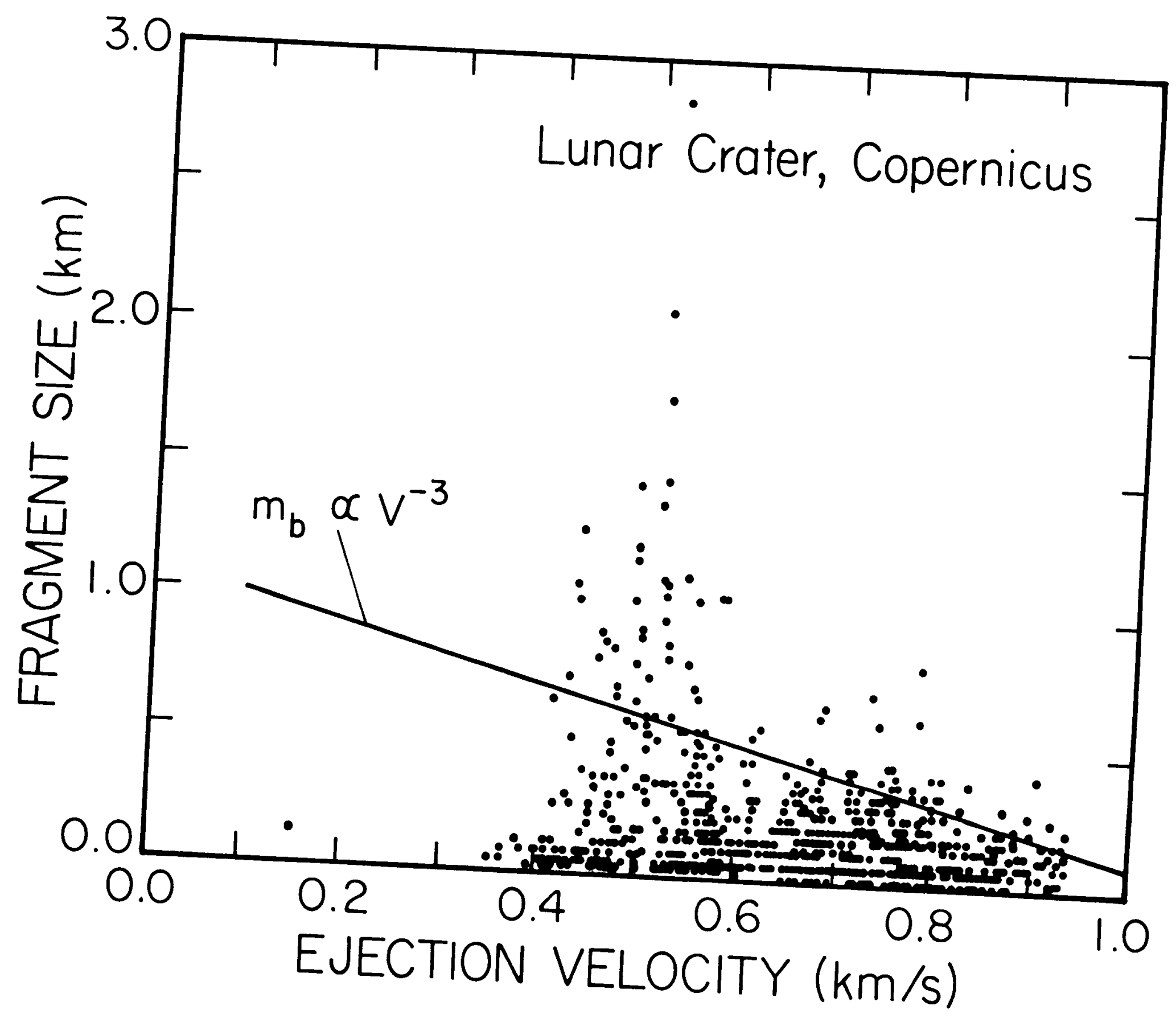




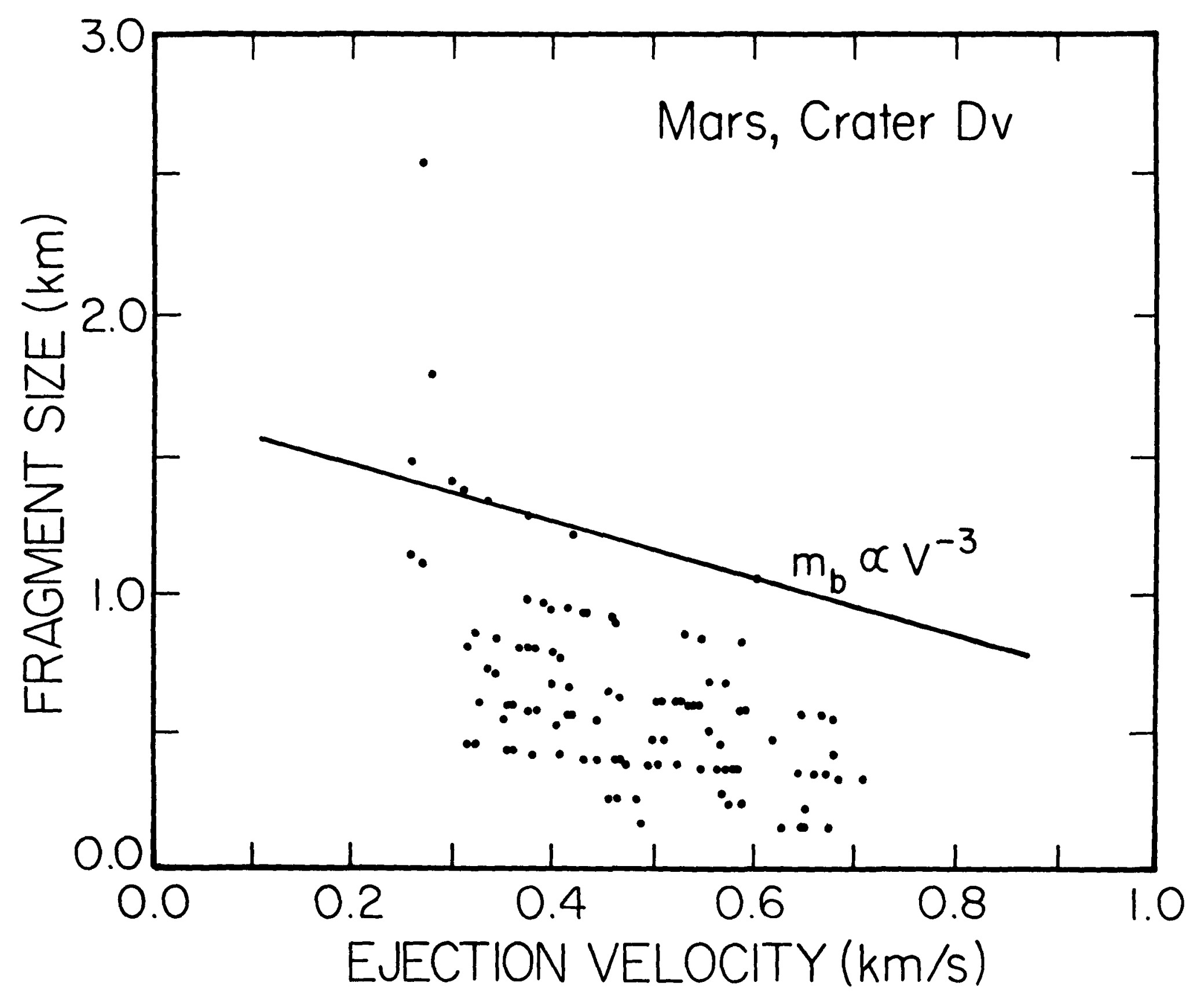




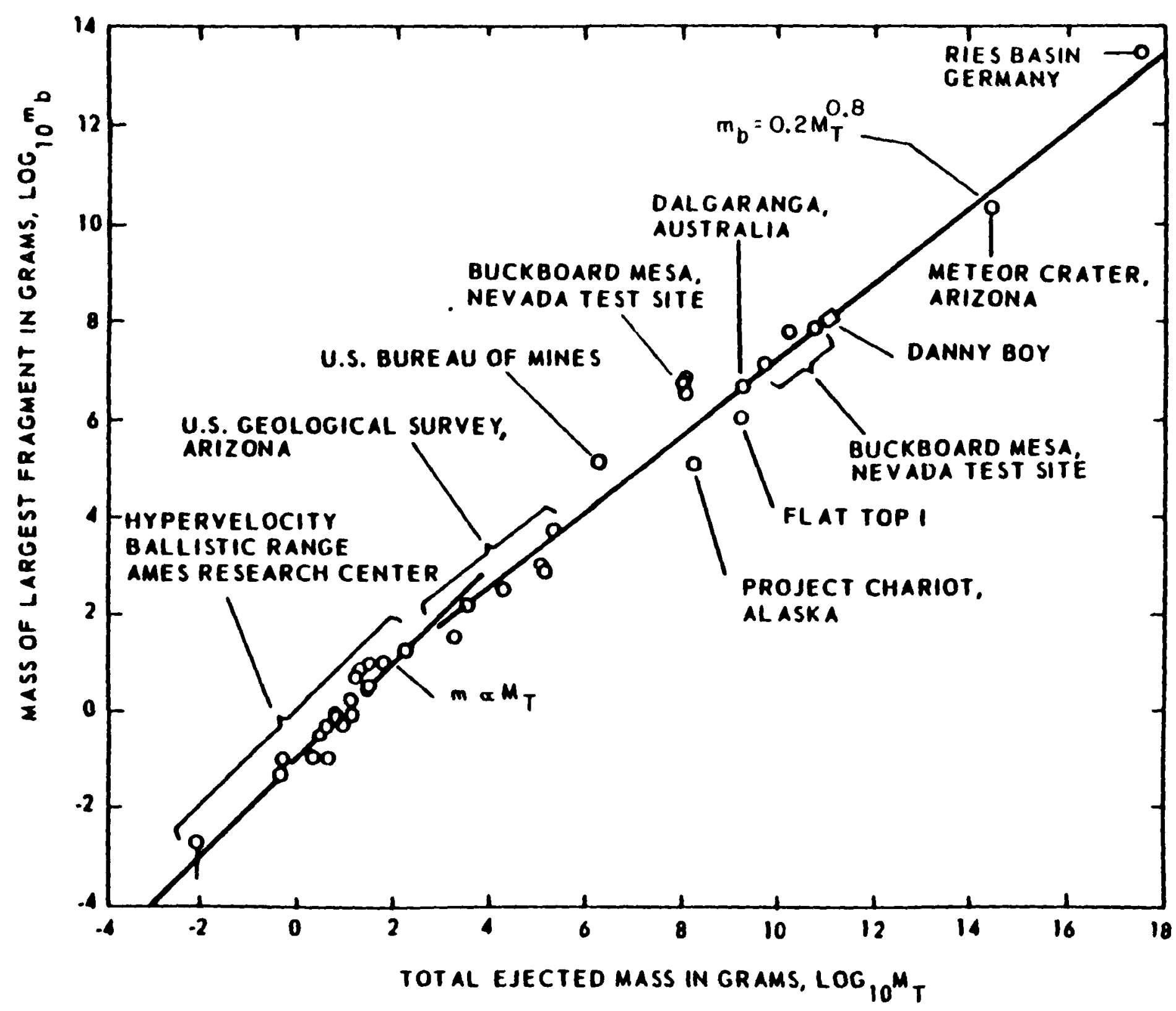




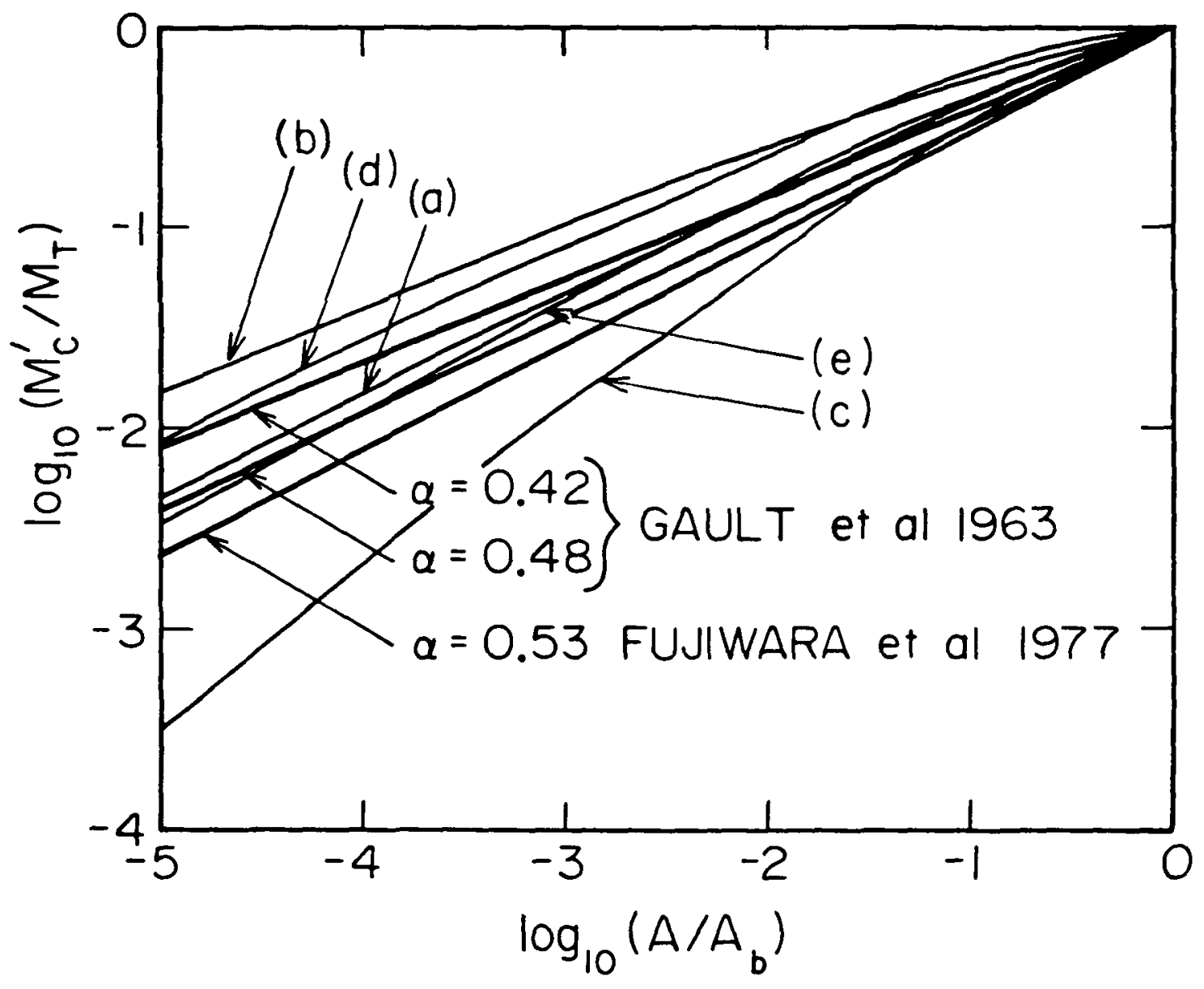

Figure 6 